\title{
Draft Genome of the Asian Buffalo Leech Hirudinaria manillensis
}

\author{
De-Long Guan ${ }^{1}$, Jie Yang ${ }^{2}$, Ying-Kui Liu ${ }^{3}$, Yuan $\mathrm{Li}^{4}$, Da Mi ${ }^{4}, \mathrm{Li}$-Bin $\mathrm{Ma}^{1}$, Zhe-Zhi Wang ${ }^{1 *}$, \\ Sheng-Quan $\mathrm{Xu}^{1 *}$ and Qiang Qiu ${ }^{2 *}$ \\ ${ }^{1}$ College of Life Sciences, Shaanxi Normal University, Xi'an, China, ${ }^{2}$ Center for Ecological and Environmental Sciences, \\ Northwestern Polytechnical University, Xi'an, China, ${ }^{3}$ College of Biomedical Sciences \& Department of Biological Sciences, \\ Xuzhou Medical University, Xuzhou, China, ${ }^{4}$ Nextomics Biosciences Institute, Wuhan, China
}

OPEN ACCESS

Edited by:

Chuan-Le Xiao,

Sun Yat-sen University, China

Reviewed by:

Jia-Tang Li,

Chengdu Institute of Biology (CAS),

China

Dong-Dong Wu,

Kunming Institute of Zoology (CAS),

China

${ }^{*}$ Correspondence:

Zhe-Zhi Wang

zzwang@snnu.edu.cn

Sheng-Quan Xu

xushengquan@snnu.edu.cn

Qiang Qiu

qiuqiang@/zu.edu.cn

Specialty section:

This article was submitted to Genomic Assay Technology,

a section of the journal

Frontiers in Genetics

Received: 18 June 2019 Accepted: 04 December 2019

Published: 16 January 2020

Citation:

Guan D-L, Yang J, Liu Y-K, Li Y, Mi D,

Ma L-B, Wang Z-Z, Xu S-Q and Qiu Q (2020) Draft Genome of the Asian Buffalo Leech Hirudinaria manillensis.

Front. Genet. 10:1321.

doi: 10.3389/fgene.2019.01321
The Asian Buffalo leech, Hirudinaria manillensis, is an aquatic sanguivorous species distributed widely in Southeast Asia. H. manillensis has long been used clinically for bloodletting and other medical purposes. Recent studies have focused on artificial culturing, strain optimization, and the identification and development new drugs based on the anticoagulant effects of $H$. manillensis bites; however, data regarding its genome remain unclear. This study aimed to determine the genome sequence of an adult Asian Buffalo leech. We generated a draft assembly of $151.8 \mathrm{Mb}$ and a N50 scaffold of 2.28 Mb. Predictions indicated that the assembled genome contained 21,005 protein-coding genes. Up to 17,865 genes were annotated in multiple databases including Gene Ontology. Sixteen anticoagulant proteins with a Hirudin or Antistasin domain were identified. This study is the first to report the whole-genome sequence of the Asian Buffalo leech, an important sanguivorous leech of clinical significance. The quality of the assembly is comparable to those of other annelids. These data will help further the current understanding of the biological mechanisms and genetic characteristics of leeches and serve as a valuable resource for future studies.

Keywords: Asian buffalo leech, Hirudinaria manillensis, sequencing, genome assembly and annotation, Hirudin/ Antistasin gene family

\section{INTRODUCTION}

The Asian Buffalo leech, Hirudinaria manillensis (NCBI taxonomy ID: 1348078) (Figure 1) is a member of family Hirudinidae, order Arhynchobdellida, and phylum Annelida. H. manillensis is a type of hermaphrodite segmented worm endemic to southeast Asia (Liu et al., 2015; Oliver and DeLoughery, 2019). The leech is widely distributed in the Philippines, Vietnam, Malaysia, and several provinces of China including the Guangxi, Hunan, and Fujian provinces (Sket and Trontelj, 2007; Liu et al., 2015).

H. manillensis displays various fascinating behavioral and physiological characteristics that are of interest to evolutionary, biochemical, and pharmaceutical studies. H. manillensis differs from

Abbreviations: BUSCO, Benchmarking Universal Single-Copy Orthologs; LTRs, terminal repeat retrotransposons; MITE, miniature inverted-repeat transposable elements; TE, transposable element; SSR, simple sequence repeat; GO, gene ontology; KEGG, Kyoto Encyclopedia of Genes and Genomes; TrEMBL, Translated EMBL Nucleotide Sequence Data Library. 


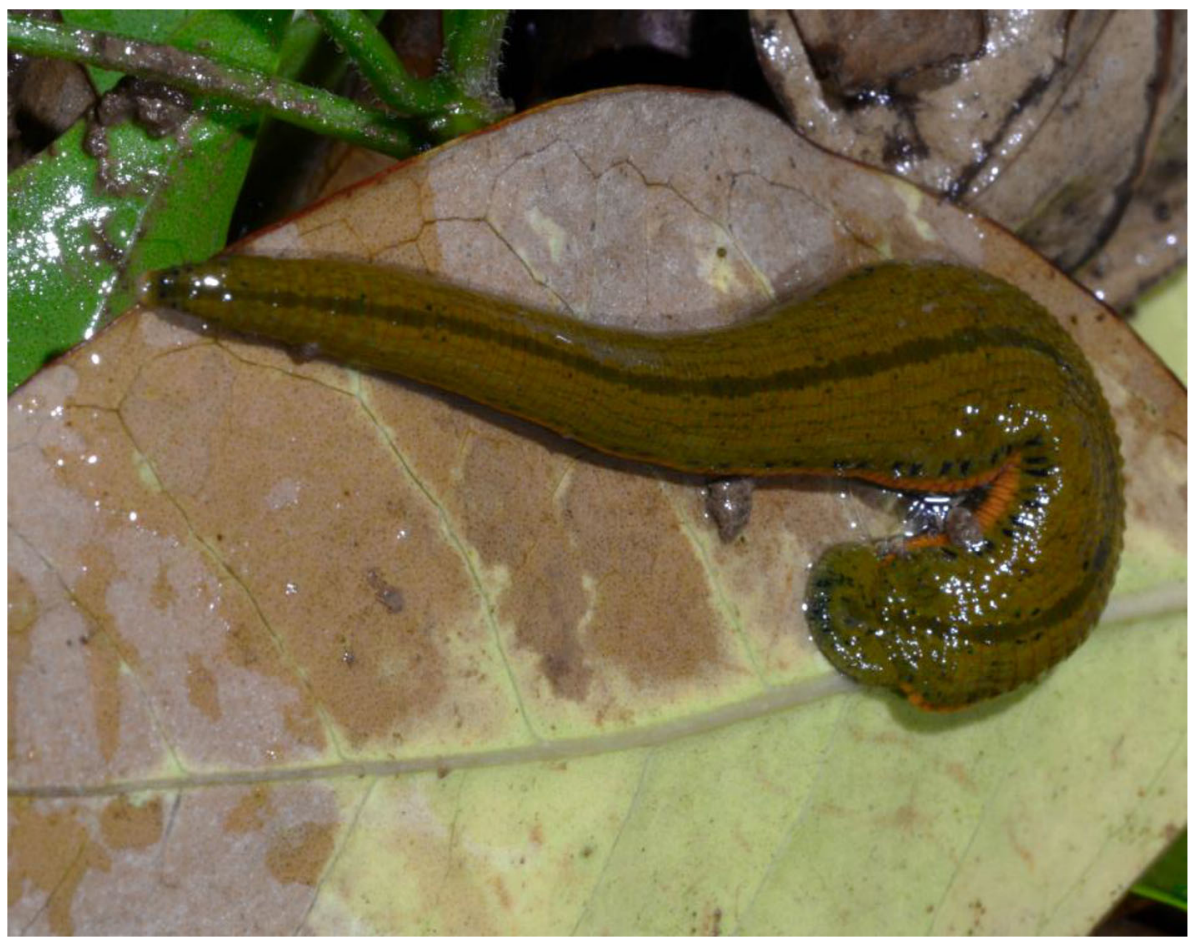

FIGURE 1 | The adult Asian Buffalo leech, Hirudinaria manillensis (image copy-right retrieved from Li-Bin Ma).

oligochaetes, which typically have 2 suckers at each end of the body, by having independent internal and external body segmentations and a robust muscle coelom; these properties make H. manillensis a good model for studying the evolution of the annelid body plan (Muller et al., 1981; Apakupakul et al., 1999; Struck et al., 2007; Teut and Warning, 2008; Oliver and DeLoughery, 2019). Moreover, H. manillensis is a sanguivorous species, which is an important behavioral aspect in leeches with clinical significance (Chiang et al., 2000; Thompson, 2010; Kvist et al., 2011; Gãdekmerdan et al., 2011). The emergence of sanguivorous behavior and efficient anti-coagulation secretions provide important insights into specific adaptions of medicinal leeches (de Koning et al., 2000; Striepen et al., 2004). The jaw of H. manillensis is tripartite and filled with hundreds of tiny, sharp teeth for attaching to mammalian hosts such as cattle, horses, and humans. Studies have described the anticoagulant effects of H. manillensis bites, as sites bleed copiously even hours after leech detachment (Elliott and Sawyer, 1987; Teut and Warning, 2008). This anticoagulant effect is considered the most important feature of $H$. manillensis and has facilitated its application for clinical bloodletting and other clinical applications (Sawyer, 1981; Abdelgabar and Bhowmick, 2003). The baked whole body of $H$. manillensis is an important ingredient in Chinese traditional medicine and is used to promote blood circulation and relieve gore (Chiang et al., 2000). Recent increases in the incidence of blood clots and stroke have led to numerous studies on the utility and market potential of $H$. manillensis products.
An increasing number of studies have focused on artificial culturing, strain optimization, and the identification and development of new drugs based on this species; nonetheless, genome and genetic data are unavailable for this species. Thus far, the genome of only one non-blood sucking leech, Helobdella robusta, has been characterized and applied for studies on its bilateral symmetry (Simakov et al., 2013). Even at the phylum level, the genome sequences of only three additional species, Capitella teleta, Hydroides elegans, and Eisenia fetida, are available (Veenstra, 2011; Zwarycz et al., 2015), and lowcoverage genomic data are available for two lineages of Amynthas cortices for microsatellite analysis (Cunha et al., 2017). Accordingly, the whole-genome characterization of $H$. manillensis would be of great value with respect to its genetic foundations and mechanisms; therefore, this study aimed to determine the genome sequence of an adult Asian Buffalo leech, Hirudinaria manillensis.

\section{METHODS}

\section{Sample Collection and Sequencing}

Genomic DNA was extracted from muscle tissue dissected from the body of a single adult Asian Buffalo leech captured in a rice field in HeChi city, Guangxi Province. This species is an invertebrate and there are no restrictions on capturing adult leeches. DNA extraction was performed using a QIAamp DNA 
extraction kit (Qiagen, Hilden, Germany) in accordance with the manufacturer specifications. In total, $20 \mu \mathrm{g}$ DNA was retrieved and used for genome sequencing.

A short-insert library was sequenced using the Hiseq 4000 platform (Illumina, San Diego, CA, USA) to generate an initial survey in accordance with the standard protocol. In detail, a part of the DNA sample was randomly fragmented using an ultrasonic fragmentation apparatus. The library was prepared in accordance with the procedure of end repair, adding adaptor, purification, and PCR amplification. In total, $12.3 \mathrm{~Gb}$ of raw reads were obtained. Through prior whole-genome sequencing, a $20 \mathrm{k}$ SMRTcell library was constructed using the standard protocol of Pacbio (Pacific Biosciences, San Diego, CA, USA). Sequencing was performed in two SMRT cells on the Pacbio sequel platform. Briefly, the DNA sample was initially trimmed using Diagenode Megaruptor2 (Luxembourg, Belgium), and universal hairpin adapters were ligated onto double-stranded DNA fragments. Adapter dimers were eliminated using Pacific Biosciences' (PacBio's) MagBead kit. Thereafter, the failed ligation DNA fragments were eliminated using exonucleases. After exonuclease and AMPure PB purification, sequencing primers were annealed to the SMRTbell templates to facilitate the binding of the sequence polymerase. In total, approximately $15.1 \mathrm{~Gb}(\sim 100 \mathrm{X})$ of long raw reads were generated. Statistics for genome sequencing are listed in Table S1 (Supplementary Data Sheets 1: Table S1).

\section{Quality Control of Sequenced Reads}

For short reads, the Trimmomatic java package (Trimmomatic, RRID: SCR 011848, Version 0.38) (Bolger et al., 2014) was used to eliminate adapter-contaminated reads (defined as matches with the adapter sequence library $>10 \mathrm{bp}$ ), low-quality or empty reads (defined as reads with $>5 \%$ undetermined bases or a Phred quality score $<30$ ). After processing, we obtained $12.2 \mathrm{~Gb}$ of clean data. Regarding the Pacbio sequel data, we obtained approximately $14.9 \mathrm{~Gb}$ of clean data after eliminating adaptercontaminated reads and low-quality reads $<500 \mathrm{bp}$, using Proovread (Version 2.12), an error correction package included in the CANU (CANU, RRID: SCR_015880, Version 2.12) (Koren et al., 2017). Overall, we retained $98.6 \%$ of the raw Pacbio sequel data including 1,505,497 reads.

\section{Nuclear Genome Assembly}

We used CANU (CANU, RRID: SCR_015880, Version 2.12) (Koren et al., 2017), LoRDEC (LoRDEC, RRID: SCR_015814, Version 0.3) (Salmela and Rivals, 2014), and MECAT (Xiao et al., 2017) packages (https://github.com/xiaochuanle/MECAT) for sequence calibration and genome assembly. This assembly process references a previous successful assembly attempt using MECAT software (Liu et al., 2019). In brief, we first used CANU to self-correct the clean Pacbio long-reads to rectify random sequencing errors (Koren et al., 2017). Thereafter, all reads were readjusted using the short reads survey data, with LoRDEC (Salmela and Rivals, 2014) to exclude the possibility of faulty bases. Finally, calibrated reads were deposited into MECAT for complete assembly.

\section{RNA Extraction, Transcriptome Sequencing, and Processing of Reads}

Muscle tissues from six additional individuals of $H$. manillensis were obtained for transcriptome sequencing to improve the findings of genome assembly and to determine gene expression levels (Denton et al., 2014). These individuals share same breeding line with the individual used for DNA extraction and was captured from the same natural population, that is the rice field in HeChi city, Guangxi Province. To determine the differentially expressed genes associated with feeding, these individuals were segregated into two groups and have undergone different treatments through starving and blood-feeding.

RNA extraction was performed using a RNeasy Mini Kit (Qiagen, Hilden, Germany) in accordance with the manufacturer's instructions. Two cDNA libraries with inserted sequence sizes of $150 \mathrm{~kb}$ were constructed for sequencing using the Hiseq 4000 platform (Illumina, San Diego, CA, USA) for transcriptome sequencing. In brief, approximately $3 \mu \mathrm{g}$ of total RNA for each sample was used to prepare RNA-seq libraries. The mRNA was enriched using poly-T oligo-attached magnetic beads from the total RNA and subsequently randomly fragmented in fragmentation buffer (Biomarker, Beijing, China). Thereafter, the fragments were used as the template for cDNA synthesis using random hexamers. Retrieved double-strand cDNA fusion was achieved and purified using AMPure XP beads (Beckman Coulter, California, USA). Finally, the final cDNA libraries with the reads of preferentially 150-200 bp were selected with the AMPure XP system. In total, raw reads of $42.81 \mathrm{~Gb}$ were obtained.

Using the RNA-seq data, a referenced genome-guided mapping against the assembly of $H$. manillensis was performed for each of the paired-end samples, using Hisat2 (HISAT2, RRID: SCR_015530) (Kim et al., 2015). The stringtie software (StringTie, RRID: SCR_016323, Version 2.0) (Pertea et al., 2015) was then used to determine the FPKM values of all genes to perform differential expression analysis of the two conditions (fasting and feeding).

\section{Genome Assembly Assessment}

All data that retrieved from Illumina sequencing were aligned to the assembled genome. The generated bam file was handled using Samtools (Samtools, RRID: SCR_002105) (Li et al., 2009) and finally used to check and adjust genome accuracy, using Pilon (Pilon, RRID: SCR_014731) (Walker et al., 2014). Furthermore, Benchmarking Universal Single-Copy Orthologs (BUSCO, RRID: SCR 015008) (Simao et al., 2015) was used to check the completeness of the genome assembly based on metazoan core genes. BUSCO was used with the metazoan orthologous gene set, and the complete, fragment, and lost genes were determined in the assembly.

\section{Determination of Repetitive Elements}

We determined repetitive sequences using RepeatMasker (RepeatMasker, RRID: SCR_012954) and RepeatModeler (RRID: SCR_015027) pipeline (Huda and Jordan, 2009). 
RepeatModeler was used to determine a consensus sequence for each repeat family. Results from RepeatModeler and Annelida repeat sequence database were combined. This combined database was used to annotate and mask repetitive sequences in the $H$. manillensis genome. The results are presented in Table S2 (Supplementary Data Sheet 2: Table S2).

\section{Gene Prediction}

We combined $A b$ initio and homology-based prediction methods to construct consensus gene models. We used PASA (PASA, RRID: SCR_014656) (Haas et al., 2003), Genscan (GENSCAN, RRID: SCR_012902) (Burge and Karlin, 1997), Augustus (Augustus: Gene Prediction, RRID: SCR 008417) (Stanke et al., 2006), GlimmerHMM (GlimmerHMM, RRID: SCR_002654) (Majoros et al., 2004), GeneID (GeneID, SCR_002473) (Alioto et al., 2018), and SNAP (Korf, 2004) to search for gene models. Using the homology-based method, protein sequences of Caenorhabditis elegans (nematode, GCA_000002985.3), Capitella teleta (Marine worm, GCA_000328365.1), and Helobdella robusta (freshwater leech, GCA_000326865.1) were downloaded from the NCBI GenBank database (GenBank, RRID: SCR_002760) (Benson et al., 2014) and implemented for library construction. The data were deposited into GeMoMa (Keilwagen et al., 2016), which is a jar package integrated into an analysis pipeline. Finally, gene models from the $A b$ initio and homology-based prediction methods were combined into a non-redundant gene set of 21,005 genes, using EVidenceModeler (EVidenceModeler (EVM), RRID: SCR_014659) software (Supplementary Data Sheet 2: gene_finding.gff) (Haas et al., 2008).

\section{Gene Function Annotation}

For functional annotation, the Pfam database (PFAM, RRID: SCR_004726) (Punta et al., 2011) was used to annotate proteins, using HMMER (HMMER, RRID: SCR_005305) (Finn et al., 2011). Gene Ontology (GO; GO, RRID: SCR 002811) (Sherlock, 2009) terms were obtained using blast2go (Blast2GO, RRID: SCR_005828) (Conesa et al., 2005). Metabolic functions of these genes were assigned using the Kyoto Encyclopedia of Genes and Genomes (KEGG, RRID: SCR 012773) database (Kanehisa and Goto, 2000). The motifs and domains of genes were determined using InterProScan (InterProScan, RRID: SCR 005829) (Zdobnov and Apweiler, 2001) against the InterProscan protein signature databases including HAMAP (HAMAP, RRID: SCR_007701) (Lima et al., 2009), PRINTS (PRINTS, RRID: SCR 003412) (Attwood et al., 2003), Pfam (Pfam, RRID: SCR 004726) (Punta et al., 2011), SMART (SMART, RRID: SCR 005026) (Letunic et al., 2011), PANTHER (PANTHER, RRID: SCR 004869) (Mi et al., 2010), and ProDom (ProDom, RRID: SCR 006969) (Servant et al., 2002). All annotations are shown in Supplementary Data Sheet 1.

\section{Data Analysis and Visualization}

Synteny analysis was conducted using MCscanX (Wang et al., 2012). GO and KEGG enrichment analyses were conducted using KOBAS 3.0 (KOBAS, RRID: SCR_006350). Tables and figures were visualized using Geneious, TBtools (Chen et al., 2018) and MS Excel.

\section{RESULTS AND DISCUSSION}

\section{Genome Sequencing and Assembly}

A high-quality draft genome of $H$. manillensis was obtained. The resulting assembly included 467 scaffolds with a total length of 151.8 Mb. A comparison between the assembled genome size with the kmer-based estimated size is a common measurement to reveal missing sequences. Using the obtained short reads, the whole genome length was approximately $160.5 \mathrm{Mb}$, suggesting that our assembly is approximately $94.5 \%$ complete with few missing sequences.

The length of scaffolds is highly variable. The present assembly displayed a scaffold N50 of $2.28 \mathrm{Mb}$ and an overall GC content of $35.98 \%$. The longest scaffold was of $5.92 \mathrm{Mb}$ and the median scaffold length was $17,504 \mathrm{bp}$. These parameters are comparable with the genome assemblies of other annelids and the most outstanding advantage for the present assembly is the marked reduction in the number of scaffolds (Table 1). The enhanced connectivity of scaffolds is reflected in the average length, which is $324,782 \mathrm{bp}$. Compared with that of H. robusta, the present scaffold is approximately 2.74 -fold longer.

Thereafter, we evaluated the completeness of the $H$. manillensis assembly by searching for 978 metazoa-conserved BUSCOs (Simao et al., 2015). All repetitive elements were pre-

TABLE 1 | Comparison of the genome assembly and number of genes with published genomes of other annelids.

\begin{tabular}{|c|c|c|c|c|c|c|c|c|}
\hline & $\begin{array}{l}\text { Genome Len } \\
\text { (bp) }\end{array}$ & Seq Num & $\begin{array}{c}\text { GC content } \\
(\%)\end{array}$ & $\begin{array}{l}\text { Minimum Len } \\
\text { (bp) }\end{array}$ & $\begin{array}{l}\text { Max Len } \\
\text { (bp) }\end{array}$ & $\begin{array}{l}\text { Mean Len } \\
\text { (bp) }\end{array}$ & $\begin{array}{l}\text { Median Len } \\
\text { (bp) }\end{array}$ & N50 (bp) \\
\hline Hirudinaria manillensis & $151,673,145$ & 467 & 0.36 & 2,693 & $5,920,317$ & 324,782 & 17,504 & $2,277,518$ \\
\hline $\begin{array}{l}\text { Helobdella robusta } \\
\text { (GCA_000326865.1) }\end{array}$ & $235,376,169$ & 1,991 & 0.33 & 1,000 & $13,640,604$ & 118,220 & 3,236 & $3,060,193$ \\
\hline $\begin{array}{l}\text { Capitella teleta } \\
\text { (GCA_000328365.1) }\end{array}$ & $333,283,208$ & 20,803 & 0.40 & 1,000 & $1,620,044$ & 16,021 & 2,479 & 188,402 \\
\hline Eisenia fetida (GCA_003999395.1) & $1,471,976,452$ & 399,003 & 0.40 & 500 & 91,445 & 3,689 & 764 & 9,314 \\
\hline Eisenia fetida (GCA_900000155.1) & $1,052,631,503$ & $1,659,527$ & 0.41 & 100 & 58,500 & 634 & 182 & 1,852 \\
\hline $\begin{array}{l}\text { Hydroides elegans } \\
\text { (GCA_001703475.1) }\end{array}$ & $1,026,046,400$ & 188,407 & 0.35 & 300 & 244,066 & 5,446 & 1,225 & 17,329 \\
\hline
\end{tabular}

Len, length; seq num, sequence number. 
masked to prevent unnecessary intervention. Consequently, 897 (91.7\%) complete BUSCOs and 20 (2.0\%) fragmented BUSCOs were identified, while only $61(6.3 \%)$ BUSCOs were missing from the assembly. Based on a previous estimation of assembly integrity, these numbers also suggested that the present assembly is approximately $93.7 \%$ (complete and fragmented BUSCOs) complete and has high quality.

Furthermore, we performed BUSCO analysis for other published annelid genomes, using the same parameters and compared the completeness of each genome assembly (Figure 2). Both assemblies of Eisenia fetida did not yield long scaffolds and the maximum length was only 91,445 and 58,500 $\mathrm{bp}$; therefore, they were excluded from further comparative genomic analysis. The quality of the $H$. manilensis assembly was comparable with that of others, and most of the determined BUSCOs (711) were shared among these four genomes. The number of identified complete BUSCOs was greater in the present assembly than in that of $H$. robusta (91.7 to $89.7 \%$ ).

\section{Determination of Repetitive Elements}

RepeatMasker and RepeatModeler pipeline analyses revealed that the $H$. manillensis assembly comprises $19.52 \%$ (29.6 Mb) of repetitive elements (Table S2). This proportion of repetitive elements was greater than that of $H$. robusta (17.33\%). Hence, some repetitive elements resembled protein-coding sequences; they were subsequently replaced use $\mathrm{N}$ to facilitate the accuracy of gene identification. In addition, by combining the genome size analysis of these two species, it was not difficult to determine that $H$. robusta has a larger genome size, but a smaller proportion of repeat sequences, contrary to previous reports regarding the structure and composition of the genome. Therefore, we speculate that the underlying reason may be the difference in sequencing technology when obtaining the genome assembly. Previously, genome acquisition of $H$. robusta was carried out through next-generation short-read-length sequencing technology. Because the length of a single read is limited to several hundred base pairs (usually 150 or $250 \mathrm{bp}$ ), the repeated fragments on the genome usually cannot be correctly interpreted, resulting in the deletion and reduction of repeat sequences in the assembly. However, with third-generation technology, its sequencing reads can approach a read length of more than 20 $\mathrm{kb}$, thus greatly enhancing its ability to span high complex repeating fragments of the genome and significantly increasing the number of identified repeat sequences and improve the quality and accuracy of the genome assembly.

\section{Gene Identification and Annotation}

Using the masked assembly, combining the $A b$ initio and homology-based prediction method, we finally identified 21,005 protein coding genes in $H$. manillensis genome, with high confidence. The total length of all genes was $62.23 \mathrm{Mb}$, representing $41.03 \%$ of the genome. The average length of genes and CDSs are 2,963.1 bp and 1,293.3 bp, respectively. Mean protein sequence length is 431.1 aa (amino acids). A BUSCO assessment was conducted to evaluate the completeness of these determined genes, revealing $90.1 \%$ complete BUSCOs. Almost all BUSCOs predicted from the genome assembly were present in the identified genes, and these gene models had high integrity.

Regarding gene annotation, 17,865 genes showed highconfidence matches (E-value $\leq 1 \mathrm{e}-5)$ in the Pfam $(14,287$ genes), GO (16,050 genes), KEGG (6,435 genes), interproscan (16,588 genes), and NCBI's non-redundant (NR) protein databases $(16,945)$, accounting for $85.05 \%$ of total number of genes. The source species of annotations retrieved from the NR

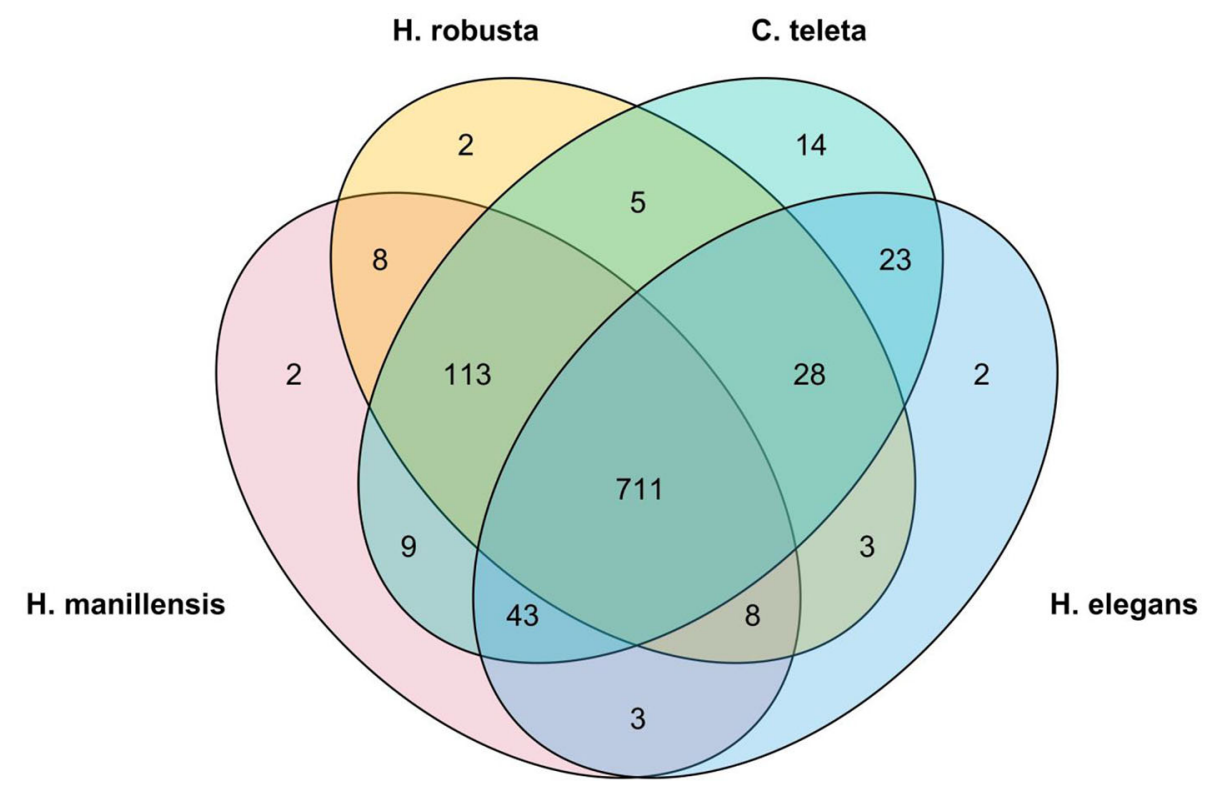

FIGURE 2 | Venn plot of the common identified BUSCOs among all four published annelids' genome assemblies. 


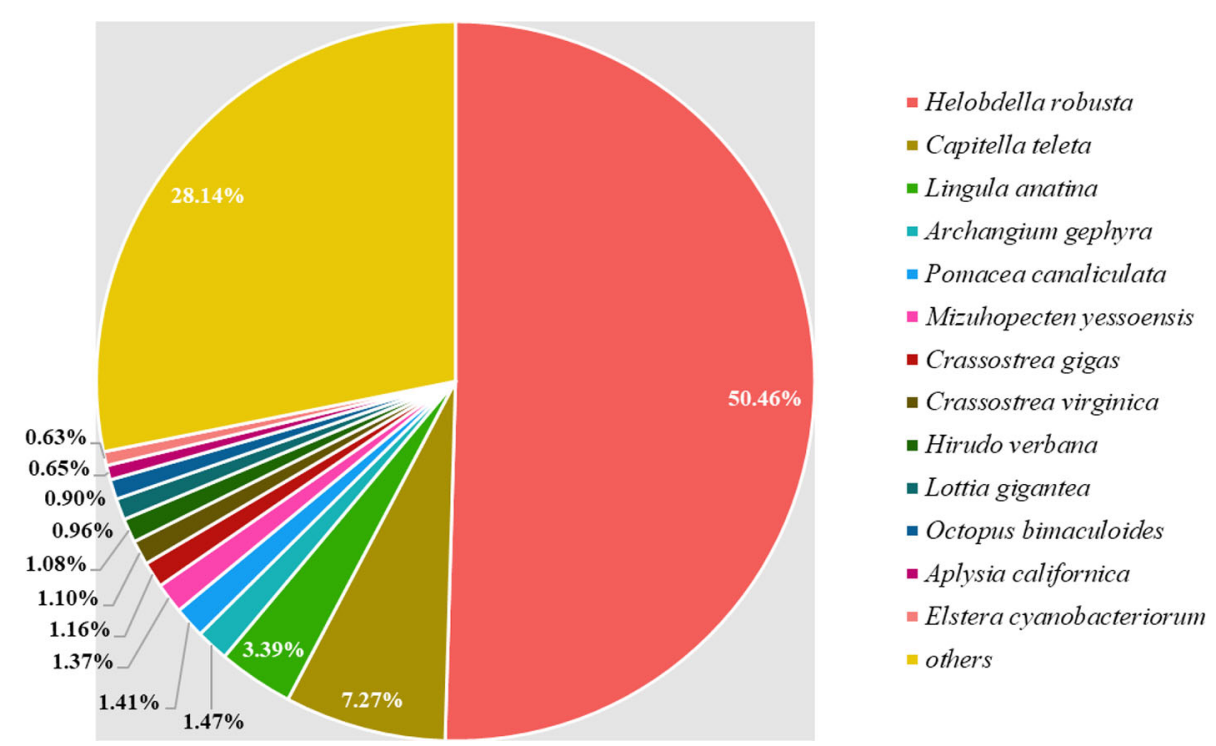

FIGURE 3 | Distribution of NR annotations retrieved from different source species. Each of the species is denoted by a different color. The "others" section in the figure represents species occupying neglectable proportions.

were traced (Figure 3), and $50.46 \%$ were known sequences from $H$. robusta, which was the best match. As species of leeches, they share similar genetic backgrounds. Furthermore, C. teleta and Lingula anatina contribute to 7.27 and $3.39 \%$ of NR annotations and are distant relatives of leeches from the superorder Lophotrochozoa.

GO is a standardized gene functional classification system, which comprehensively describes the properties of genes and gene products in organisms. We classified all the retrieved GO terms and found that biological processes related to vital metabolic pathways were most enriched in terms of cellular processes, biological regulation, response to stimuli, etc. (Figure 4).

\section{Intraspecific/Interspecific Synteny}

Intraspecific/interspecific synteny analysis was performed using MCscanX (Figure 5A, B), revealing 246 syntenic gene blocks (in 123 pairs), representing $1.17 \%$ of the total protein coding

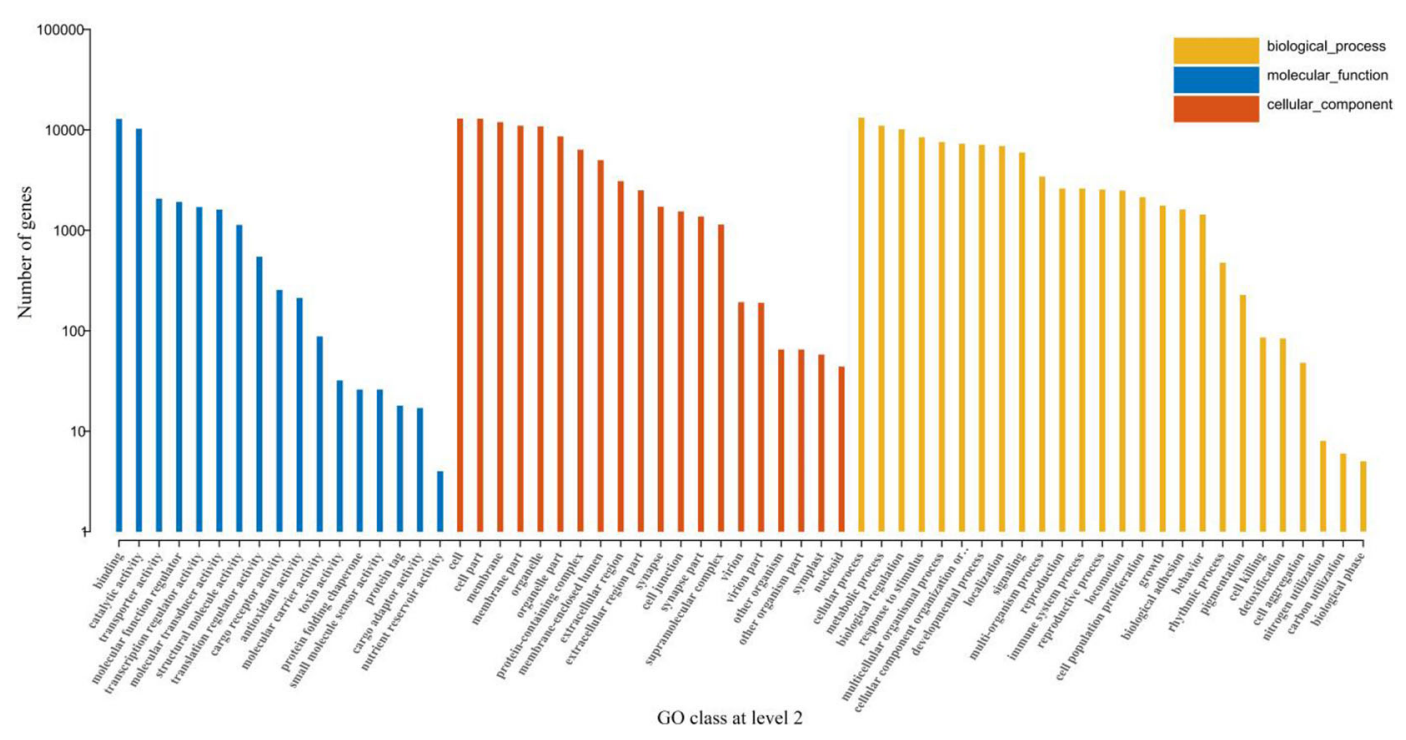

FIGURE 4 | GO classification at level 2. The lateral-axis shows the GO terms, while the vertical-axis shows the number of genes accounted for in each term. The blue, red, and orange bars represent different GO classifications of the molecular function, cellular component, and biological process, respectively. 


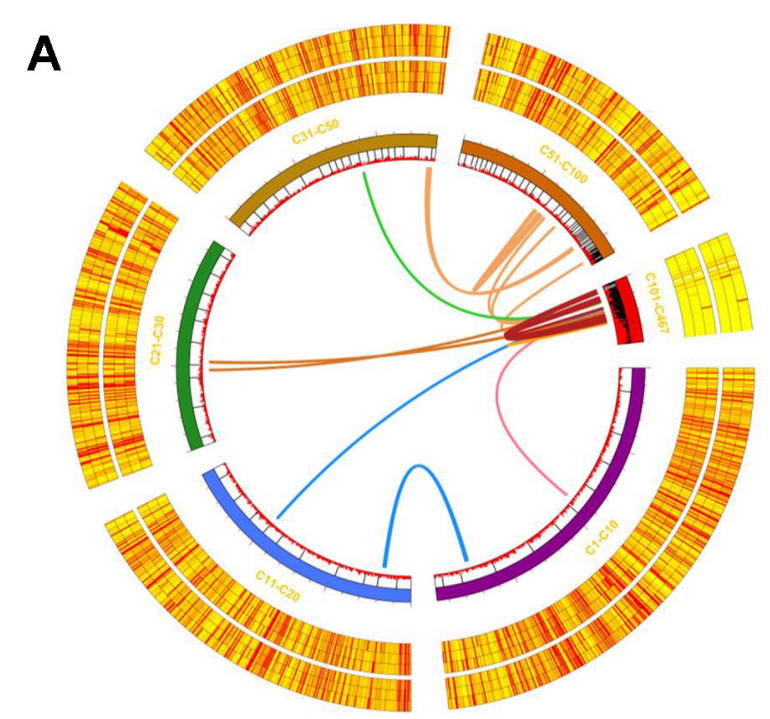

B

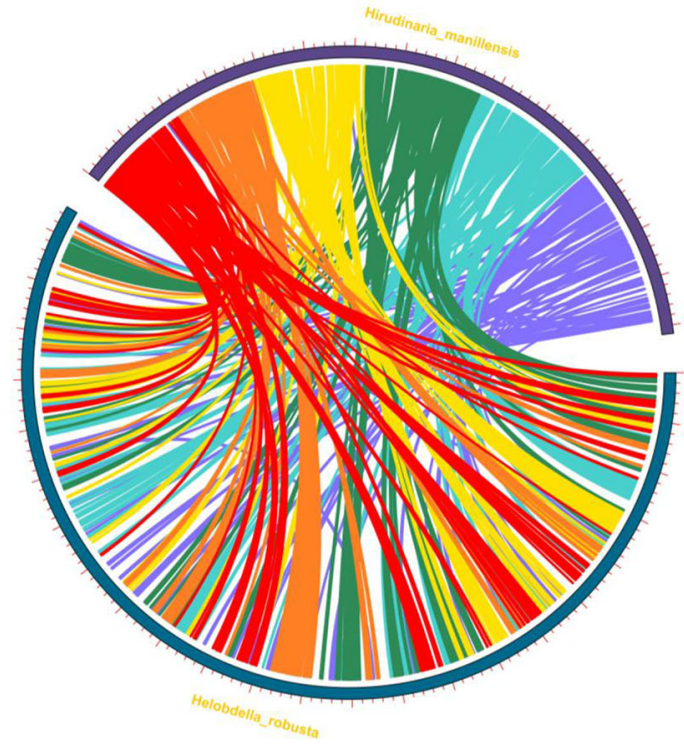

FIGURE 5 | Intraspecific/interspecific MCscanX synteny analyses. (A) Wholegenome syntenic mapping of $H$. manillensis genes to itself. The innermost lines represent synteny linkage; the innermost circle represents the gene distribution, and the tagged circles (C1-C10, C11-C20, C21-C30, C31-C50, C51-C100,C101-C467) represent different merged contigs; the outsider heatmap circles represent the expression level (six samples, before and after feeding, from inner to outside). (B) Circular view of the synteny between the $H$. manillensis genes and the $H$. robusta genes.

sequences (Figure 5A). The H. manillensis genome has few intraspecific collinear genes, indicating that genome-wide gene duplication has a limited effect during long-term evolution in this species. Moreover, the interspecific gene synteny linkage between the genome of $H$. manillensis and $H$. robusta revealed
9,383 interspecific syntenic gene blocks (21.12\% of all genes) of 4,724 pairs among them (Figure 5B). These two species share genome-wide syntenic linkage, suggesting that they are genetically related and have a common ancestor.

\section{Genetic Implications for Anticoagulant Proteins}

The ability to synthesize anticoagulant substances is the most valuable feature for $H$. manillensis. We therefore examined the composition of anticoagulant proteins, focusing on those with two Pfam protein domains: Hirudin (Pfam entry: PF00713) and Antistasin (Pfam entry: PF02822), which are the functional fundamental inhibitors of thrombin. To our knowledge, almost all known anticoagulant proteins in leeches contain such domains, as in NCBI Identical Protein Groups, and their members include Hirudin (most famous natural thrombin inhibitor), antistasin (inhibitor of blood coagulation factor $\mathrm{Xa}$ ), hirustasin (inhibitor of tissue kallikrein, trypsin, alphachymotrypsin, and granulocyte cathepsin G), bdellin or bdellastasin (inhibitor of trypsin, plasmin, and acrosin), and decorsin (inhibitor of platelet aggregation).

As in $H$. manillensis, 16 proteins contained either the Hirudin or Antistasin domain. According to their annotations, most of them resembled the commonly known anticoagulants in leeches including Hirudin (Swiss-prot ID: Q07558), Guamerin (Swiss-prot ID: P46443), and Bdellastasin (Swiss-prot ID: P82107). However, one gene of the Antistasin family (evm.model.Contig00032.80) was different from any known protein in the NR or Swiss-prot database; hence, we inferred that it is a novel protein potentially resulting from intense gene recombination and may have different functions. Further, to determine the presence of this gene encoding this protein, we determined its expression levels, and the average FPKM value was 11.92 among six samples.

Moreover, while analyzing these anticoagulant proteins, we noticed an interesting pattern in a gene cluster on Contig 6 . There were seven linearly distributed genes: from evm.model.Contig00006.200 to evm.model.Contig00006.206, having conserved sequences encoding Guamerin (except for evm.model.Contig00006.201) (Figure 6). Multiple gene duplications were expected for these genes, along with a greater requirement of Guamerin, an elastase-specific inhibitor.

\section{CONCLUSION}

This study is the first to present the annotated genome sequence assembly of the Asian Buffalo leech. We generated a draft longread genome assembly of $151.8 \mathrm{~Gb}$ and a N50 scaffold size of 2.28 $\mathrm{Mb}$. The assembled genome was predicted to contain 21,005 protein-coding genes and up to $29.6 \mathrm{Mb}$ (17.3\%) of repetitive elements. In total, 17,865 genes were annotated. Genes encoding anticoagulants such as Hirudin were present and a gene cluster encoding Guamerin was identified. This annotated draft genome for $H$. manillensis provides valuable data for captive leech breeding and facilitates further studies on the phylogeny and 


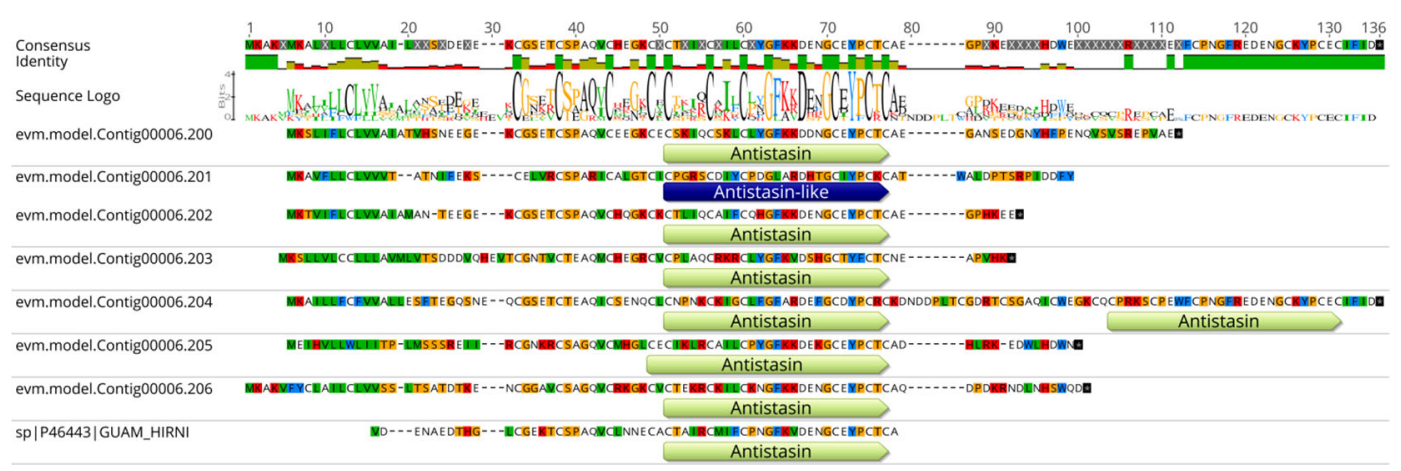

FIGURE 6 | The distribution of Antistasin domains in the gene cluster on contig00006 of the assembly of $H$. manillensis. The gene IDs are shown on the left. The consensus sequence of the alignment is shown on the top of the figure. After the identity of the sequences and the sequence logo, the variations among these sequences are shown.

biological features of $H$. manillensis, such as the biosynthesis of anticoagulant proteins and genetic adaptions for blood sucking (Abdelgabar and Bhowmick, 2003; Struck et al., 2007; Thompson, 2010; Struck et al., 2011).

\section{DATA AVAILABILITY STATEMENT}

Raw data have been deposited in NCBI SRA (sequence Read Archive) database with the project accession PRJNA475873 (genome) and PRJNA477611 (survey). The SRA accessions are SRR7415780 (genome) and SRR7415797 (survey), respectively. The RNA sequence dataset supporting the present results is available in the repository of NCBI Sequence Read Archive (SRA) with the following accession Number: SRX6035209 $\sim$ SRX6035214.

\section{ETHICS STATEMENT}

Mature H. manillensis samples were collected from ponds in Hechi City, Guangxi Province, China. As prescribed by "Law of People's Republic of China on the Protection of Wildlife" and "Regulations for the Implementation of the People's Republic of China on the Protection of terrestrial Wildlife" (State Council Decree [1992] No. 13), specimen collection did not require any ethical or institutional approval because $H$. manillensis is not endangered or protected by any current law. The care and

\section{REFERENCES}

Abdelgabar, A. M., and Bhowmick, B. K. (2003). The return of the leech. Int. J. Clin. Pract. 57 (2), 103-105. doi: 10.1016/0140-6736(92)92110-2

Alioto, T., Blanco, E., Parra, G., and Guigó, R. (2018). Using geneid to Identify Genes. Curr. Protoc. Bioinformatics 64, e56. doi: 10.1002/cpbi.56

Apakupakul, K., Siddall, M. E., and Burreson, E. M. (1999). Higher level relationships of leeches (Annelida: Clitellata: Euhirudinea) based on treatment of animals in this study were in accordance with the Guideline for the Care and Use of Laboratory Animals in China.

\section{AUTHOR CONTRIBUTIONS}

D-LG, S-QX, and QQ designed the study. L-BM, Y-KL, and ZZW collected the samples. DM and YL conducted the sequencing analysis. D-LG and JY were involved in the data analyses. D-LG, JY, and S-QX wrote the manuscript. All authors read and approved the final manuscript.

\section{FUNDING}

This study was financially supported by the Excellent Doctor Innovation Project of Shaanxi Normal University (S2015YB03), the Fundamental Research Funds for the Central Universities (GK201903063; GK201702010), the National Natural Science Foundation of China (No.31872273), and the Key Research and Development Program of Shaanxi Province (2018ZDXMSF-079).

\section{SUPPLEMENTARY MATERIAL}

The Supplementary Material for this article can be found online at: https://www.frontiersin.org/articles/10.3389/fgene.2019. 01321/full\#supplementary-material

morphology and gene sequences. Mol. Phylogenet. Evol. 12 (3), 350. doi: 10.1006/mpev.1999.0639

Attwood, T. K., Bradley, P., Flower, D. R., Gaulton, A., Maudling, N., Mitchell, A. L., et al. (2003). PRINTS and its automatic supplement, prePRINTS. Nucleic Acids Res. 31 (1), 400-402. doi: 10.1093/nar/gkg030

Benson, D. A., Clark, K., Karsch-Mizrachi, I., Lipman, D. J., Ostell, J., and Sayers, E. W. (2014). GenBank. Nucleic Acids Res. 42, D32-D37. doi: 10.1093/nar/ gkt1030 
Bolger, A. M., Lohse, M., and Usadel, B. (2014). Trimmomatic: a flexible trimmer for Illumina sequence data. Bioinformatics 30 (15), 2114. doi: 10.1093/ bioinformatics/btu170

Burge, C., and Karlin, S. (1997). Prediction of complete gene structures in human genomic DNA. J. Mol. Biol. 268 (1), 78-94. doi: 10.1006/jmbi.1997.0951

Chen, C., Xia, R., Chen, H., and He, Y. (2018). TBtools, a Toolkit for Biologists integrating various HTS-data handling tools with a user-friendly interface. bioRxiv 289660. doi: 10.1101/289660

Chiang, Y. C., Yang, C. K., Shih, J. C., and Lee, C. N. (2000). The Pharmacopoeia of the People's Republic of China. (English edition). Can. J. Behav. Sci. 26 (4), 534-550.

Conesa, A., Götz, S., Garcíagómez, J. M., Terol, J., Talón, M., and Robles, M. (2005). Blast2GO: a universal tool for annotation, visualization and analysis in functional genomics research. Bioinformatics 21 (18), 3674. doi: 10.1093/ bioinformatics/bti610

Cunha, L., Thornber, A., Kille, P., Morgan, A. J., and Novo, M. (2017). A large set of microsatellites for the highly invasive earthworm Amynthas corticis predicted from low coverage genomes. Appl. Soil Ecol. 119, 152-155. doi: 10.1016/j.apsoil.2017.05.029

de Koning, A. P., Brinkman, F. S., Jones, S. J., and Keeling, P. J. (2000). Lateral gene transfer and metabolic adaptation in the human parasite Trichomonas vaginalis. Mol. Biol. Evol. 17 (11), 1769-1773. doi: 10.1093/oxford journals.molbev.a026275

Denton, J. F., Lugo-Martinez, J., Tucker, A. E., Schrider, D. R., Warren, W. C., and Hahn, M. W. (2014). Extensive error in the number of genes inferred from draft genome assemblies. PloS Comput. Biol. 10 (12), e1003998. doi: 10.1371/ journal.pcbi.1003998

Elliott, J., and Sawyer, R. (1987). Leech biology and behaviour-review. J. Anim. Ecol. 56, 720. doi: 10.2307/5083

Finn, R. D., Clements, J., and Eddy, S. R. (2011). HMMER web server: interactive sequence similarity searching. Nucleic Acids Res. 39 (Web Server issue), W29W37. doi: 10.1002/cpbi.40

Gãdekmerdan, A., Arusan, S., Bayar, B., and SaäŸLam, N. (2011). Medicinal leeches and hirudotherapy. Turkiye Parazitol. Derg. 35 (4), 234-239. doi: 10.5152/tpd.2011.60

Haas, B. J., Delcher, A. L., Mount, S. M., Wortman, J. R., Smith, R. K., Hannick, L. I., et al. (2003). Improving the Arabidopsis genome annotation using maximal transcript alignment assemblies. Nucleic Acids Res. 31 (19), 5654-5666. doi: 10.1093/nar/gkg770

Haas, B. J., Salzberg, S. L., Zhu, W., Pertea, M., Allen, J. E., Orvis, J., et al. (2008). Automated eukaryotic gene structure annotation using EVidenceModeler and the Program to Assemble Spliced Alignments. Genome Biol. 9 (1), R7. doi: 10.1186/gb-2008-9-1-r7

Huda, A., and Jordan, I. K. (2009). Analysis of transposable element sequences using CENSOR and RepeatMasker. Methods Mol. Biol. 537 (537), 323-336. doi: 10.1007/978-1-59745-251-9_16

Kanehisa, M., and Goto, S. (2000). KEGG: kyoto encyclopedia of genes and genomes. Nucleic Acids Res. 28, 27-30. doi: 10.1093/nar/28.1.27

Keilwagen, J., Wenk, M., Erickson, J. L., Schattat, M. H., Grau, J., and Hartung, F. (2016). Using intron position conservation for homology-based gene prediction. Nucleic Acids Res. 44 (9), e89-e89.

Kim, D., Langmead, B., and Salzberg, S. L. (2015). HISAT: a fast spliced aligner with low memory requirements. Nat. Methods 12 (4), 357-360. doi: 10.1038/ nmeth.3317

Koren, S., Walenz, B. P., Berlin, K., Miller, J. R., Bergman, N. H., and Phillippy, A. M. (2017). Canu: scalable and accurate long-read assembly via adaptive k-mer weighting and repeat separation. Genome Res. 27 (5), 722. doi: 10.1101/ gr.215087.116

Korf, I. (2004). Gene finding in novel genomes. BMC Bioinf. 5 (1), 59. doi: 10.1186/ 1471-2105-5-59

Kvist, S., Sarkar, I. N., and Siddall, M. E. (2011). Genome-wide search for leech antiplatelet proteins in the non-blood-feeding leech $\mathrm{H}$ elobdella robusta (Rhyncobdellida: Glossiphoniidae) reveals evidence of secreted anticoagulants. Invertebrate Biol. 130 (4), 344-350. doi: 10.1111/j.17447410.2011.00238.x

Letunic, I., Doerks, T., and Bork, P. (2011). SMART 7: recent updates to the protein domain annotation resource. Nucleic Acids Res. 40 (Database issue), 302-305. doi: 10.1093/nar/gkr931
Li, H., Handsaker, B., Wysoker, A., Fennell, T., Ruan, J., Homer, N., et al. (2009). The Sequence Alignment/Map format and SAMtools. Bioinformatics 25 (16), 2078-2079. doi: 10.1093/bioinformatics/btp352

Lima, T., Auchincloss, A. H., Coudert, E., Keller, G., Michoud, K., Rivoire, C., et al. (2009). HAMAP: a database of completely sequenced microbial proteome sets and manually curated microbial protein families in UniProtKB/Swiss-Prot. Nucleic Acids Res. 37 (Database issue), 471-478. doi: 10.1093/nar/gkn661

Liu, F., Guo, Q. S., Shi, H. Z., Cheng, B. X., Lu, Y. X., Gou, L., et al. (2015). Genetic variation in Whitmania pigra, Hirudo nipponica and Poecilobdella manillensis, three endemic and endangered species in China using SSR and TRAP markers. Gene 579 (2), 172-182. doi: 10.1016/j.gene.2015.12.055

Liu, Q., Fang, L., Yu, G., Wang, D., Xiao, C. L., and Wang, K. (2019). Detection of DNA base modifications by deep recurrent neural network on Oxford Nanopore sequencing data. Nat. Commun. 10 (1), 2449. doi: 10.1038/ s41467-019-10168-2

Majoros, W. H., Pertea, M., and Salzberg, S. L. (2004). TigrScan and GlimmerHMM: two open source ab initio eukaryotic gene-finders. Bioinformatics 20 (16), 2878-2879. doi: 10.1093/bioinformatics/bth315

Mi, H., Dong, Q., Muruganujan, A., Gaudet, P., Lewis, S., and Thomas, P. D. (2010). PANTHER version 7: improved phylogenetic trees, orthologs and collaboration with the Gene Ontology Consortium. Nucleic Acids Res. 38 (Database issue), 204-210. doi: 10.1093/nar/gkp1019

Muller, K. J., Nicholls, J. G., and Stent, G. S. (1981). Neurobiology of the leech [1981].

Oliver, J. D., and DeLoughery, E. P. (2019). Leeches and plastic surgery in the modern era. Plast. Surg. Nurs. 39, 95-96. doi: 10.1097/PSN.0000000000000272

Pertea, M., Pertea, G. M., Antonescu, C. M., Chang, T. C., Mendell, J. T., and Salzberg, S. L. (2015). StringTie enables improved reconstruction of a transcriptome from RNA-seq reads. Nat. Biotechnol. 33 (3), 290-295. doi: $10.1038 /$ nbt.3122

Punta, M., Coggill, P., Eberhardt, R., Mistry, J., Tate, J., Boursnell, C., et al. (2011). The Pfam protein families database. Nucleic Acids Res. 40, D290-D301. doi: 10.1093/nar/gkr1065

Salmela, L., and Rivals, E. (2014). LoRDEC: accurate and efficient long read error correction. Bioinformatics 30 (24), 3506. doi: 10.1093/bioinformatics/btu538

Sawyer, R. T. (1981). Why we need to save the medicinal leech. Oryx 16 (2), 165168. doi: $10.1017 /$ S0030605300017142

Sayers, E. W., Agarwala, R., Bolton, E. E., Brister, J. R., Canese, K., Clark, K., Connor, R., Fiorini, N., Funk, K., Hefferon, T., et al. (2019). Database resources of the National Center for Biotechnology Information. Nucleic Acids Res. 47, D23-D28. doi: 10.1093/nar/gky1069

Servant, F., Bru, C., Carrère, S., Courcelle, E., Gouzy, J., Peyruc, D., et al. (2002). ProDom: automated clustering of homologous domains. Briefings Bioinf. 3 (3), 246. doi: $10.1093 / \mathrm{bib} / 3.3 .246$

Sherlock, G. (2009). Gene Ontology: tool for the unification of biology. Can. Inst. Food Sci. Technol. J. 22 (4), 415.

Simakov, O., Marletaz, F., Cho, S. J., Edsingergonzales, E., Havlak, P., Hellsten, U., et al. (2013). Insights into bilaterian evolution from three spiralian genomes. Nature 493 (7433), 526. doi: 10.1038/nature11696

Simao, F. A., Waterhouse, R. M., Ioannidis, P., Kriventseva, E. V., and Zdobnov, E. M. (2015). BUSCO: assessing genome assembly and annotation completeness with single-copy orthologs. Bioinformatics 31 (19), 3210-3212. doi: 10.1093/ bioinformatics/btv351

Sket, B., and Trontelj, P. (2007). Global diversity of leeches (Hirudinea) in freshwater (Netherlands; Springer ).

Stanke, M., Keller, O., Gunduz, I., Hayes, A., Waack, S., and Morgenstern, B. (2006). AUGUSTUS: ab initio prediction of alternative transcripts. Nucleic Acids Res. 34 (Web Server issue), 435-439. doi: 10.1093/nar/gkl200

Striepen, B., Pruijssers, A. J., Huang, J., Li, C., Gubbels, M. J., Umejiego, N. N., et al. (2004). Gene transfer in the evolution of parasite nucleotide biosynthesis. Proc. Natl. Acad. Sci. U. S. A. 101 (9), 3154-3159. doi: 10.1073/pnas.0304686101

Struck, T. H., Schult, N., Kusen, T., Hickman, E., Bleidorn, C., Mchugh, D., et al. (2007). Annelid phylogeny and the status of Sipuncula and Echiura. BMC Evol. Biol. 7 (1), 57. doi: 10.1186/1471-2148-7-57

Struck, T. H., Paul, C., Hill, N., Hartmann, S., Hösel, C., Kube, M., et al. (2011). Phylogenomic analyses unravel annelid evolution. Nature 471 (7336), 95. doi: 10.1038 /nature09864 
Teut, M., and Warning, A. (2008). Leeches, phytotherapy and physiotherapy in osteo-arthrosis of the knee-a geriatric case study. Forschende Komplementrmedizin 15 (5), 269-272. doi: 10.1016/j.eujim.2008.08.032

Thompson, C. A. (2010). New direct thrombin inhibitor becomes available. Am. J. Health-System Pharm. 67 (8), 588-588. doi: 10.2146/news100027

Veenstra, J. A. (2011). Neuropeptide evolution: neurohormones and neuropeptides predicted from the genomes of Capitella teleta and Helobdella robusta. Gen. Comp. Endocrinol. 171 (2), 160-175. doi: 10.1016/ j.ygcen.2011.01.005

Walker, B. J., Abeel, T., Shea, T., Priest, M., Abouelliel, A., Sakthikumar, S., et al. (2014). Pilon: an integrated tool for comprehensive microbial variant detection and genome assembly improvement. PloS One 9 (11), e112963. doi: 10.1371/ journal.pone.0112963

Wang, Y., Tang, H., Debarry, J. D., Tan, X., Li, J., Wang, X., et al. (2012). MCScanX: a toolkit for detection and evolutionary analysis of gene synteny and collinearity. Nucleic Acids Res. 40 (7), e49-e49. doi: 10.1093/nar/ gkr1293

Xiao, C. L., Chen, Y., Xie, S. Q., Chen, K. N., Wang, Y., Han, Y., et al. (2017). MECAT: fast mapping, error correction, and de novo assembly for singlemolecule sequencing reads. Nat. Methods 14 (11), 1072-1074. doi: 10.1038/ nmeth. 4432
Zdobnov, E. M., and Apweiler, R. (2001). InterProScan - an integration platform for the signature-recognition methods in InterPro. Bioinformatics 17 (9), 847848. doi: 10.1093/bioinformatics/17.9.847

Zwarycz, A. S., Nossa, C. W., Putnam, N. H., and Ryan, J. F. (2015). Timing and Scope of Genomic Expansion within Annelida: Evidence from Homeoboxes in the Genome of the Earthworm Eisenia fetida. Genome Biol. Evol. 8 (1), 271. doi: 10.1093/gbe/evv243

Conflict of Interest: Authors YL and DM were employed at Nextomics Biosciences.

The remaining authors declare that the research was conducted in the absence of any commercial or financial relationships that could be construed as a potential conflict of interest.

Copyright (c) 2020 Guan, Yang, Liu, Li, Mi, Ma, Wang, Xu and Qiu. This is an openaccess article distributed under the terms of the Creative Commons Attribution License (CC BY). The use, distribution or reproduction in other forums is permitted, provided the original author(s) and the copyright owner(s) are credited and that the original publication in this journal is cited, in accordance with accepted academic practice. No use, distribution or reproduction is permitted which does not comply with these terms. 